sition but markedly change at the main transition within the very narrow temperature range of a few degrees. ${ }^{16}$

In the present DDPC-water system, on the other hand, the role of each group in the respective transitions is not so clear as in the case of the DMPC-water system. However, the comparison of the transition temperatures of DDPC with those of DMPC and the inspection of Figures 2 and 6 make the following points clear. If we consider the fact that the temperature of the transition I of DDPC with the amide group $\left(16^{\circ} \mathrm{C}\right)$ is higher than that of the pretransition of DMPC with the ester group $\left(11^{\circ} \mathrm{C}\right)$ and that the amide II band of DDPC exhibits a distinct peak at the transition I in Figure 6, this transition seems to be triggered by a loosening of the hydrogen-bonding network through the amide groups. It may induce the chain melting (see Figure 2) as well as the structural changes of the head groups as seen in Figure 6.

The temperature of the transition II agrees with that of the main transition of the DMPC-water system. As is also clear from Figure 6, all parts of DDPC are subjected

(16) Mendelsohn; R.; Dluhy, R.; Taraschi, T.; Cameron, D. G.; Mantsch, H. H. Biochemistry 1982, 38, 175 . to the structural changes to the same extent at this transition.

Finally, at the transition III, the structural changes of the phosphate and choline groups mainly occur. Possibly, the hydrogen-bonding network among the amide agroups still persists at this transition, because in DMPC, which is free from the amide group, the structural changes of head groups have already finished at lower temperatures.

In conclusion, the introduction of the amide group in DDPC gives rise to different structural aspects of the thermotropic phase transitions from those of DMPC with the ester group. First, for the $44 \mathrm{~g} \%$ DDPC- $56 \mathrm{~g} \%$ water system, three phase transition take place between 16 and $28^{\circ} \mathrm{C}$. Second, the chain melting takes place in the wide range of temperature, including three transitions, and is completed at $35^{\circ} \mathrm{C}$. Third, the structural changes of the head groups occur at the highest temperature transition III.

Acknowledgment. We thank Professor Emeritus S. Seki and Dr. M. Kodama of Kwansei Gakuin University for their kind offer of unpublished DSC data of DDPC.

Registry No. DDPC, 108861-07-0.

\title{
Orientation of Molecular Monolayers at the Liquid-Liquid Interface As Studied by Optical Second Harmonic Generation
}

\author{
S. G. Grubb ${ }^{\dagger}$ and Mahn Won Kim* \\ Exxon Research and Engineering Company, Route 22 East, Annandale, New Jersey 08801 \\ Th. Rasing and Y. R. Shen \\ Department of Physics, University of California, Center for Advanced Materials, Lawrence \\ Berkeley Laboratory, Berkeley, California 94720
}

Received May 15, 1987. In Final Form: August 17, 1987

\begin{abstract}
The molecular orientation of surfactants has been determined at air/water, decane/water, and water/carbon tetrachloride interfaces by optical second harmonic generation. The results show that the molecular orientation depends on the nature of the nonaqueous phase.
\end{abstract}

The study of molecular monolayers at the liquid-liquid interface has attracted attention from researchers in a variety of disciplines. ${ }^{1}$ Interface-active monolayers are of interest in studying interfacial mass transfer and micellar structure, and as model membrane structures. Monolayers at the interface between two immiscible liquids have been extensively studied by classical techniques such as surface tension and surface potential measurements. However, there has been a noticeable lack of information at the molecular level at the liquid-liquid interface. A few studies have used total internal reflection excited fluorescence ${ }^{2}$ and resonance Raman scattering ${ }^{3-5}$ of dye molecules at the liquid-liquid interface. In this paper we report the use of optical second harmonic generation (SHG) to determine the orientation of monolayers of sodium 1-dodecylnaphthalene-4-sulfonate (SDNS) $\left(\mathrm{C}_{12} \mathrm{H}_{25^{*}}\right.$ $\mathrm{C}_{10} \mathrm{H}_{6} \mathrm{SO}_{3} \mathrm{Na}$ ) at the aqueous/decane and aqueous/carbon tetrachloride interfaces.

'Present address: Amoco Research Center, Physical Technology Division, P.O. Box 400, Naperville, IL 60506.
Optical techniques possess the obvious advantage of being able to probe interfaces between dense media, but generally they do not exhibit the surface specificity characteristic of the electron spectroscopies. Optical second harmonic generation (SHG), however, is intrinsically surface-specific as it is forbidden in centrosymmetric media but necessarily allowed at a surface or interface. ${ }^{6}$ In addition, it shares the usual advantages of optical probes such as high spatial and temporal resolution.

The surface nonlinear susceptibility $\overline{\bar{\chi}}_{\mathrm{s}}^{(2)}$ arising from a monolayer of adsorbates can be written as $^{7-9}$

$$
\chi_{\mathbf{s}, i j k}^{(2)}=N_{\mathbf{s}}\left\langle T_{i j k}^{\lambda \mu \nu}\right\rangle \alpha_{\lambda \mu \nu}^{(2)}
$$

(1) Hanna, G. J.; Noble, R. D. Chem. Rev. 1995, 85, 583.

(2) Morrison, L. E, Weber, G., to be published

(3) Takenaka, T. Nakanaga, T. J. Phys. Chem 1976, 80, 475

(4) Nakanaga, T.; Takenaka, T. J. Phys. Chem. 1977, 81, 645.

(5) Takenaka, T. Chem. Phys. Lett. 1978, 55, 515.

(6) Shen, Y. R. J. Vac. Sci. Technol. 1985, 133, 1464.

(7) Heinz, T. F.; Tom, H. W. K.; Shen, Y. R. Phys. Rev. A 1983, 28 1883

1988 American Chemical Society 

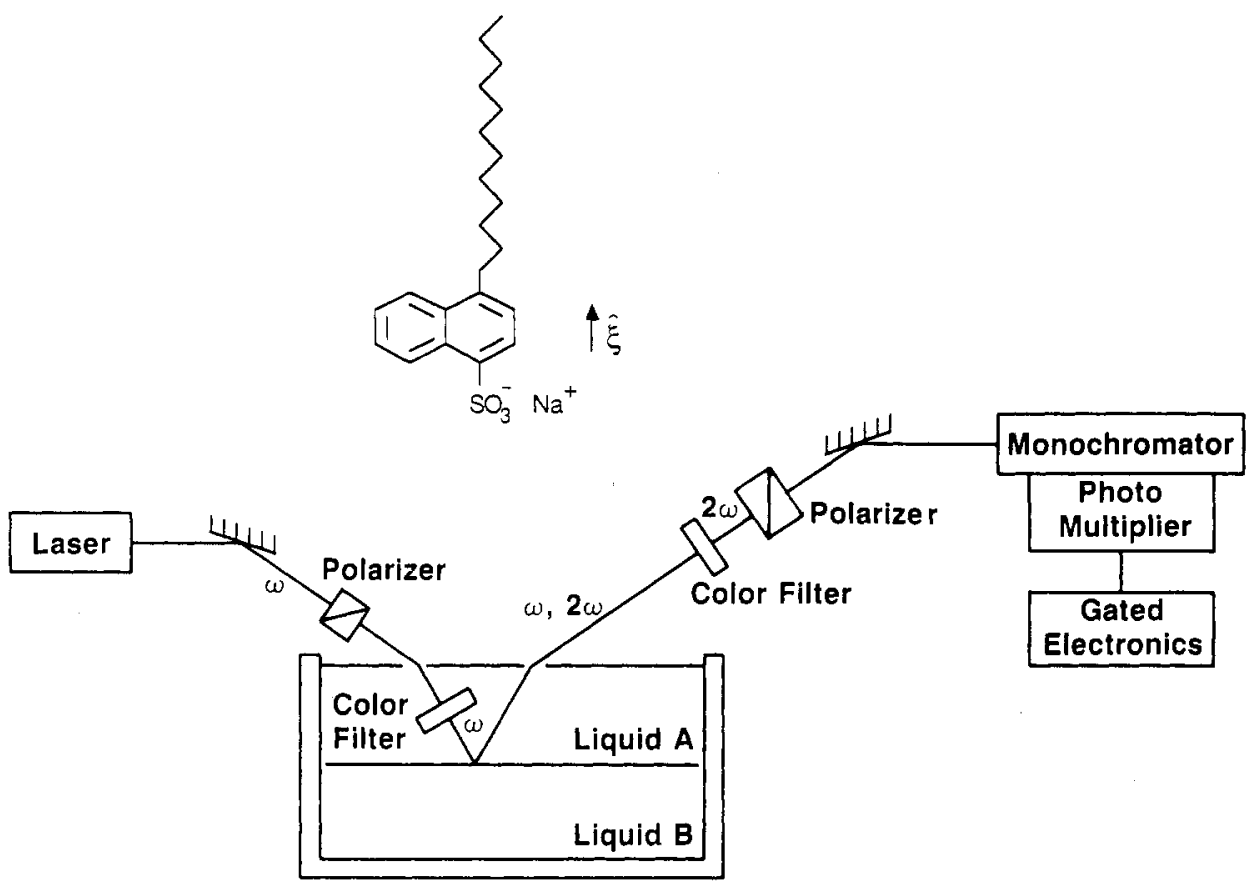

Figure 1. Schematic picture of the experimental apparatus used to observe SHG from monolayers at a liquid/liquid interface and the structure of the SDNS molecule studied.

where $N_{\mathrm{a}}$ is the surface density of molecules, $\overrightarrow{\bar{\alpha}}^{(2)}$ is the molecular nonlinear polarizability, $\left\langle T_{i j k}^{\lambda \mu \nu}\right\rangle$ is the coordinate transformation connecting the laboratory $(x, y, z)$ and molecular $(\xi, \eta, \zeta)$ axes, and the angular brackets ( ) denote an average over the molecular orientations. From the SHG measurements, we can obtain $\chi_{\mathbf{s}, i j k}^{(2)}$, but to find the molecular orientation, we need to know $\left\langle T_{i j k}^{\lambda \mu \nu}\right\rangle$, which is generally difficult to deduce. The analysis becomes tractable when $\overline{\bar{\alpha}}^{(2)}$ is dominated by a single component $\alpha_{\xi \xi \xi}^{(2)}$ along a specific molecular axis $\hat{\xi}$, which is randomly oriented with respect to the surface normal. In this case the nonvanishing elements of $\overrightarrow{\bar{\chi}}_{\mathrm{B}}^{(2)}$ are $^{7-9}$

$$
\begin{gathered}
\chi_{\mathrm{s}, z z z}^{(2)}=N_{\mathrm{s}}\left\langle\cos ^{3} \theta\right\rangle \alpha_{\xi \xi \xi}^{(2)} \\
\chi_{i \mathrm{~s}, z i i}^{(2)}=\chi_{\mathrm{s}, i z i}^{(2)}=\chi_{\mathrm{s}, l i z}^{(2)}=1 / 2 N_{\mathrm{s}}\left\langle\sin ^{2} \theta \cos \theta\right\rangle \alpha_{\xi \xi \xi}^{(2)}
\end{gathered}
$$

where $\theta$ is the polar angle between the $\hat{\xi}$ axis and the surface normal $\hat{z}$. A measurement of the ratio between any two linear combinations of $\chi_{z z z}^{(2)}$ and $\chi_{z x x}^{(2)}$ can yield a weighted average of $\theta$.

For the SHG experiments we used the frequency-doubled output of a Q-switched Nd:YAG laser at $532 \mathrm{~nm}$ as the pump beam with an energy of $15-20 \mathrm{~mJ} /$ pulse in a cross sectional area of $0.4 \mathrm{~cm}^{2}$. The $\mathrm{SH}$ signal from the interface was sufficiently weak to necessitate the use of gated photon counting techniques. There was a nonnegligible signal from the bare interface proportional to $|\overrightarrow{\tilde{\chi}}(2)-i|^{2}$ while the interface with surfactant present gave a signal proportional to $\left|\overrightarrow{\bar{\chi}}_{l-l}^{(2)}+\overrightarrow{\bar{\chi}}_{\mathbf{s}}^{(2)}\right|^{2}$. The phase difference between $\overline{\bar{\chi}}_{l-l}(2)$ and $\overrightarrow{\bar{\chi}}_{\mathrm{s}}^{(2)}$ was found to be negligible by a quartz interference technique. ${ }^{8}$

Figure 1 shows a schematic picture of the experimental apparatus and the structure of the molecule. The different $\overline{\bar{\chi}}_{s}^{(2)}$ components could be selectively measured by appropriate combinations of input and output polarizers. Possible polarization rotations at the air-liquid interfaces

(8) Rasing, Th.; Shen, Y. R.; Kim, M. W.; Valint, P., Jr.; Bock, J. Phys. Rev. A 1985, 31, 537 .

(9) Rasing, Th.; Berkovic, G.; Shen, Y. R.; Grubb, S. G.; Kim, M. W. Chem. Phys. Lett. 1986, 130, 1 . must be taken into account. A color filter was placed in the input beam inside the liquid above the liquid-liquid interface in order to block the $\mathrm{SH}$ signal generated at the air-liquid interface. Another color filter was used in the output beam to separate the $\mathrm{SH}$ signal from the fundamental after its emergence from the liquid.

The SDNA surfactant was introduced by replacing by pipette approximately $10 \mathrm{~mL}$ of the aqueous phase $(2 \%$ $\mathrm{NaCl}$ ) with a saturated $2 \% \mathrm{NaCl}$ solution of SDNS. The total aqueous concentration of SDNS was made to be approximately $10^{-5} \mathrm{M}$, which by our independently measured Langmuir adsorption isotherms was shown to lead to a condensed monolayer at the interface. Measurement of the surface pressure $\pi$ versus surface area per molecule $A$ at the air-water interface shows that the SDNS monolayer only shows an appreciable surface pressure close to a full monolayer and that there the area per molecule hardly changes ( $A \simeq 45 \AA^{2}$, see ref 8 ). Therefore, although we have not measured the $\pi-A$ diagrams at the liquidliquid interface, we assume that in all cases $A \simeq 45 \AA^{2}$ for a fully condensed monolayer and use the surface pressure as an indication that this latter state has been reached.

Solvent purity was determined to be critical in these measurements. Both decane and carbon tetrachloride were from Burdick \& Jackson Lab., Inc., spectroscopic grade with UV cutoffs at 193 and $261 \mathrm{~nm}$, respectively. A lower grade solvent gave rise to a broad two-photon excited luminescence background, which even with extensive spatial filtering could interfere with the measurement of the SHG.

It has been shown that the nonlinear polarizability tensor of SDNS is dominated by $\alpha_{\xi \xi \xi}^{(2)}$ arising from the naphthalene chromophore. ${ }^{9}$ Given the structure of the molecule (see Figure 1), the nonlinearity is expected to originate mostly from the asymmetry induced in the $\pi$ electron system of the naphthalene by the presence of the $\mathrm{SO}_{3}$ group on one side and the hydrocarbon chain on the other side. Based on these considerations, we assign the molecular $\hat{\xi}$-axis with the naphthalene chromophore in the way as indicated in Figure 1. The angle $\theta$ deduced from our SHG experiments, assuming a $\delta$-function orientational distribution, then will correspond to the orientation of this naphthalene portion with respect to the surface normal. 
Table I. Values of the Average Polar Angle from the Surface Normal, $\theta$, for a SDNS Monolayer at Several Interfaces $^{a}$

\begin{tabular}{ccccc}
\hline interface & $\theta$ & $\epsilon(0)$ & $\begin{array}{c}\sigma_{\mathrm{B}} \text {, dyn } / \mathrm{cm} \\
\text { (without } \\
\text { monolayer) }\end{array}$ & $\begin{array}{c}\sigma_{\mathrm{B}}, \mathrm{dyn} / \mathrm{cm} \\
\text { (with } \\
\text { monolayer) }\end{array}$ \\
\hline air $/ \mathrm{H}_{2} \mathrm{O}$ & 13 & 1.0 & 72 & 21.5 \\
$\mathrm{C}_{10} \mathrm{H}_{22} / \mathrm{H}_{2} \mathrm{O}$ & 21 & 1.991 & 50.1 & 12.3 \\
$\mathrm{H}_{2} \mathrm{O} / \mathrm{CCl}_{4}$ & 38 & 2.238 & 45 & 7.5
\end{tabular}

a The aqueous phase in all cases is $2 \mathrm{wt} \% \mathrm{NaCl} . \sigma_{\mathrm{B}}=$ interfacial tension; $\sigma_{\mathrm{s}}=$ interfacial tension in presence of SDNS monolayer; $\epsilon(0)$ is the low-frequency dielectric constant of the nonaqueous phase.

(In the following, whenever we mention the experimentally obtained molecular orientation, we are referring to this naphthalene portion.)

Whereas the polar $\mathrm{SO}_{3}$ group will be in contact with the aqueous phase, the hydrocarbon chains are expected to be more or less normal to the interface. However, given the area per molecule $\left(A \sim 45 \AA^{2}\right)$, they have a considerable degree of freedom and can be quite disordered.

Table I lists the value of $\theta$ obtained for fully condensed monolayers at several interfaces. The results of $\theta=13^{\circ}$, $21^{\circ}$, and $38^{\circ}$ for air/water, ${ }^{10}$ decane/water, and water/ carbon tetrachloride, respectively, show that the orientation of SDNS molecules strongly depends on the nature of the materials forming the interface. The orientation of a surfactant molecule is expected to depend on the ratio between the size of the hydrophilic and hydrophobic part (HLB), ${ }^{11}$ the molecule-molecule interaction, and the molecule-substrate interaction. The HLB of a molecule is mainly determined by its chemical structure. ${ }^{12}$

Recent theoretical studies show that a decrease in the tail-tail interaction will lead to an increase in the molecular tilt angle. ${ }^{11}$ The tail-tail interaction could be reduced by increasing the low-frequency dielectric constant $\epsilon(0)$ of the nonaqueous phase (see Table I). The tail-tail interaction could also be affected by the interaction between the hydrophobic parts and the nonaqueous phase. Decane easily mixes with the hydrocarbon tails but not with naphthalene. Because carbon tetrachloride is known to interact with naphthalene,$^{13}$ this may affect the interaction between the hydrophobic parts even more. If these interactions of the hydrophobic tails with the nonaqueous phase lead to an effective decrease in the tail-tail interaction, this could qualitatively explain the observed variation in the tilt angle

(10) The difference of polar angle of SDNS molecules at the air/water interface with our previous result ( $r$ f 8 ) is due to the selection of a dielectric constant of the monolayer. Since we have determined the origin of the SHG, we use 2.5 for the dielectric constant for the monolayer at all the interfaces instead of 1.0 as in ref 8.

(11) Carlson, J., to be published.

(12) Robbins, M. L. Micellization, Solubilization, and Micromulsion Mittal, K. L., Ed.; Plenum: New York, 1977.

(13) Birks, J. B. Photophysics of Aromatic Molecules; Wiley-Interscience: New york, 1970. of SDNS at air/water, decane/water, and water/carbon tetrachloride interfaces.

Molecular dynamics simulations of the structure of monolayers show that the molecules tend to tilt more if the interface is less sharp. ${ }^{14}$ The root mean square of the interfacial thickness is inversely proportional to the interfacial tension. It is interesting to note that our observed increase in $\theta$ is indeed parallel with a decrease in the interfacial tension $\sigma_{\mathrm{B}}$ (see Table I).

As has been stated before, our results for $\theta$ only refer to the naphthalene portion of the SDNS molecule. A more complete picture could be given if one would also know the structure of the hydrocarbon tails. Due to their negligible contribution of the nonlinearity, ${ }^{9}$ SHG measurements will not be able to address this part of the problem. However, by using another interface-specific $\overrightarrow{\bar{\chi}}^{(2)}$ process like infrared-visible sum-frequency generation, one could obtain the orientation of the hydrocarbon chains by studying the vibrational spectrum (the $\mathrm{C}-\mathrm{H}$ modes) of the monolayer. ${ }^{15}$

In conclusion, we have demonstrated that the technique of optical second harmonic generation can be used to determine the molecular orientation of monolayers at a liquid-liquid interface. In the case of sodium 1-dodecylnaphthalene-4-sulfonate at a water/nonaqueous interface, we find that the molecular orientation of surfactants depends strongly on the nature of the nonaqueous phase. Such information is very important for the understanding of the formation of microemulsions ${ }^{16}$ and other surfactant-related liquids such as foams. More detailed studies are needed to establish more concrete correlations between the molecular orientation and the zero-frequency dielectric constant, interfacial tension, and solubility. Future investigations will also have to include molecular orientational changes due to changing surface molecular density, aqueous electrolyte concentration, and monolayer phase transitions. ${ }^{17,18}$ By exploiting the intrinsically fast time scale of pulsed lasers, SHG can also be utilized to follow kinetics at a liquid-liquid interface.

Acknowledgment. Part of this work was supported by the Director, Office of Energy Research, Office of Basic Energy Sciences, Materials Sciences Division of the U.S. Department of Energy under Contract No. DE-AC0376SF00098.

Registry No. SDNS, 111844-14-5; $\mathrm{CCl}_{4}, 56-23-5$; decane, 124-18-5.

(14) Adin Mann, J, et al., to be published.

(15) Hunt, J. H.; Guyot-Sionnest, P.; Shen, Y. R. Chem. Phys. Lett. 1987, 133, 189.

(16) Safran, S. A. Surfactants in Solution; Mittal, K. L., Lindman, B., Eds.; Plenum: New York, 1984

(17) Rasing, Th.; Shen, Y. R.; Kim, M. W.; Grubb, S. Phys. Rev. Lett. $1985,55,2903$

(18) Takenada, T.; Isono, N.; Unemura, J.; Shimomura, M.; Kunitake, T. Chem. Phys. Lett. 1986, 128, 551. 